\title{
MedienPädagogik
}

Zeitschrift für Theorie und Praxis der Medienbildung

www.medienpaed.com

ISSN 1424-3636

Jahrbuch Medienpädagogik 17:

Lernen mit und über Medien in einer digitalen Welt

Herausgegeben von Klaus Rummler, Ilka Koppel, Sandra Aßmann,

Patrick Bettinger und Karsten D. Wolf

\section{Zwischen traditionellem Schulbuch und hybridem Lehrmittel}

\section{Bedingungen, Möglichkeiten und Herausforderungen von Lernen und Lehren in einer digitalen Welt}

Alexandra Totter, Julia Häbig, Daniela Müller-Kuhn und Enikö ZalaMezö

\section{Zusammenfassung}

Der Einsatz digitaler Medien wird auch im Schulkontext immer wichtiger. Lernen und Lehren über und mit digitalen Medien wird gefordert. Die Umsetzung beinhaltet jedoch nicht nur Chancen, sondern auch technologische und organisationale Herausforderungen. Hier setzt der Beitrag an: Am Beispiel eines hybriden Französischlehrmittels, bei dem digitale Medien integrales Element des Lehrmittels sind, werden während des ersten Jahres der Einführung die Praktiken und Erfahrungen aufgezeigt. Die Perspektive der Schulleitung, der Lehrpersonen und der Schülerinnen und Schüler wird erhoben und die damit verbundenen Möglichkeiten und Herausforderungen für das Lernen und Lehren mit digitalen Medien aufgezeigt. 


\title{
Between traditional textbook and hybrid educational media. Conditions, opportunities and challenges of learning and teaching in a digital world
}

\begin{abstract}
The use of digital media is becoming increasingly important in the school context. Learning as well as teaching about and with digital media is demanded. However, the realization not only involves opportunities, but also technological and organizational challenges. This is where the contribution starts: Using the example of a hybrid French textbook, in which digital media are integral element of the textbook, the practices and experiences are examined during the first year of implementation. The perspectives of the school leader, teachers and pupils are surveyed and the associated opportunities and challenges for learning and teaching with digital media are highlighted and discussed.
\end{abstract}

\section{Vom Schulbuch zum hybriden Lehrmittel}

Dem Lehrmittel bzw. Schulbuch wird eine grosse Bedeutung für das Lernen und Lehren zugeschrieben, da es das dominierende Medium ist, das sowohl von Lehrpersonen als auch von Schülerinnen und Schülern in einem Unterrichtsfach über einen längeren Zeitraum verwendet wird (Hiller 2012). Sie haben eine inhaltliche, normative als auch kommunikative Funktion: «Lehrmittel [...] können über ihre symbolische Darstellungsweise verbindend-konstruierend zum Wissensaufbau beitragen oder auch hemmend Barrieren bilden und so Lernprozesse behindern» (Heitzmann und Niggli 2010, 9 f.). Lehrmittel zeichnen sich grundsätzlich als «Medien mit didaktisch mehr oder weniger präparierten inhaltlichen Aussagen, die Lernenden den Zugang zum Gegenstand erleichtern» (Heitzmann und Niggli 2010, 11) aus.

Sowohl in der Medienpädagogik als auch in der Schulbuchforschung wird seit einigen Jahren der Einsatz von digitalen Medien im (Fremdsprachen-)Unterricht diskutiert (Astleitner 2012; Matthes, Schütze, und Wiater 2013). Aktuell erfolgt eine Auseinandersetzung über die Entwicklung 
digitaler Lehrmittel (Döbeli Honegger, Hielscher, und Hartmann 2018). Macgilchrist (2017) unterscheidet dabei drei Formen digitaler Lehrmittel:

- PDF-Digitalisate, die im Prinzip ein PDF des gedruckten Lehrmittels sind.

- Multimedial-interaktive digitale Lehrmittel, die «als digitale Produkte konzipiert und entwickelt (born-digital) [werden]» (ebd. 227).

- Konstruktiv-interaktive digitale Lehrmittel, die so angelegt sind, dass Schülerinnen und Schüler selbst Inhalte erstellen oder verändern können.

Allerdings finden sich nur wenige Beispiele zum Einsatz digitaler Lehrmittel, z.B. das Mathematiklehrmittel «MeBook» (Mader und Bachinger 2017) oder «digi4schools» respektive das Projekt «E-Books in Action» (Sankofi 2017).

Döbeli Honegger et al. (2018) weisen darauf hin, dass die Digitalisierung von Lehrmitteln als Prozess in Stufen verstanden werden muss, der in vielen Fällen nie ein 100\% digitales Lehrmittel zum Ziel hat. So entstehen zurzeit als eine Mischform sogenannte hybride Lehrmittel. Diese bestehen aus einem gedruckten Buch bzw. traditionellen Lehrwerksteilen, in das digitale Medien mit multimedial-interaktiven Elementen integriert sind. Solch ein hybrides Lehrmittel zeichnet sich als ein Medienverbundsystem aus, welches sich auf unterschiedliche Symbolsysteme zum Veranschaulichen und Sichtbarmachen von Lerninhalten stützt.

Trotz der durchschnittlichen Verfügbarkeit einer digitalen Infrastruktur verzeichnete die Schweiz in der PISA Umfrage 2018 (Konsortium PISA.ch 2019) einen geringeren schulischen Einsatz digitaler Medien als der OECD-Durchschnitt. Mit der Umsetzung des Lehrplans 21 (Deutschschweizer Erziehungsdirektoren-Konferenz 2016) werden derzeit neue hybride Lehrmittel entwickelt und laufend eingeführt. Ein aktuelles Beispiel dafür ist das hybride Französischlehrmittel «dis donc!» (Egli u.a. 2017).

Der Frage, wie ein hybrides Lehrmittel von Schülerinnen und Schülern als auch Lehrpersonen tatsächlich genutzt wird und mit welchen Möglichkeiten und Herausforderungen die Einführung und der Einsatz in Schulen einhergeht, wird in diesem Artikel nachgegangen. 


\subsection{Funktion und Nutzung von Lehrmitteln}

Wie bereits eingangs erwähnt, haben Lehrmittel eine Reihe von Funktionen. In der Literatur wird insbesondere auf die didaktische Funktion von Lehrmitteln verwiesen (Hacker 1980; Hiller 2012; Fuchs, Niehaus, und Stoletzki 2014; Mader und Bachinger 2017; Heitzmann und Niggli 2010), die weiter differenziert werden kann in:

- Strukturierung: Das Lehrmittel gibt eine grundlegende Struktur und Lernfelder vor. Der Inhalt eines Faches wird in Themen unterteilt und in eine bestimmte Reihenfolge gebracht. Es fungiert als Planungshilfe für die Lehrperson.

- Repräsentation: Im Lehrmittel wird ein bestimmter Sachverhalt durch Bilder und Texte repräsentiert. Materialien zur Erarbeitung von Themen werden zur Verfügung gestellt.

- Steuerung: Das Lehrmittel bietet didaktische Steuerungselemente wie Impulse, Fragen, Aufforderungen und Arbeitsanweisungen.

- Motivierung: Im Lehrmittel sind Themen und Sachverhalte so dargestellt, dass sie Lernanreize für die Schülerinnen und Schüler schaffen.

- Differenzierung: Im Lehrmittel werden vielfältige Lernangeboten zur Verfügung gestellt, um Differenzierung zu ermöglichen.

- Übung und Kontrolle: Übungsaufgaben, Merkhilfen und Lernerfolgskontrollen sind im Lehrmittel enthalten.

In jüngster Zeit werden diese Funktionen um eine weitere, nämlich die Innovationsfunktion von Lehrmitteln ergänzt (Fuchs, Niehaus, und Stoletzki 2014). Diese geht mit der Erwartung einher, Veränderungen der methodischen und didaktischen Gestaltung des Unterrichts aber auch gesellschaftliche Wandlungsprozesse in den Unterricht zu transferieren (Schmidt 2019). Durch die Entwicklung hybrider Lehrmittel und die Integration digitaler Medien ergeben sich unter anderem folgende Veränderungen (Schaumburg 2015; Schulze-Vorberg u.a. 2018; Rodríguez Regueira und Rodríguez Rodríguez 2015):

- Vernetzte multimedial und interaktiv aufbereitete Informationen bzw. Inhalte.

- Computervermittelte synchrone und asynchrone Kommunikation.

- Gemeinsames Entwickeln und Gestalten digitaler Produkte mittels netzbasierter Arbeitsumgebungen. 
- Individuelle Lernunterstützung bzw. selbstregulierte Lernmöglichkeiten durch adaptive und adaptierbare digitale Medien und formative Assessments.

- Implizite Schulung von Anwendungskompetenz durch Office-Anwendungen.

Obwohl dem Einsatz hybrider Lehrmittel im Schulkontext Potenziale zugeschrieben werden, finden sich in der Literatur auch Hinweise darauf, dass dieser mit gewissen Herausforderungen einhergeht. Ein zentraler Aspekt dieser Herausforderungen ist die digitale Infrastruktur in Schulen (Schulze-Vorberg u.a. 2018; Gerick u.a. 2014). Ebenso stellt für Schülerinnen und Schüler die Verfügbarkeit zu Hause eine grundsätzliche Voraussetzung dar (Totter und Wolfer 2016).

Beim Einsatz hybrider Lehrmittel im Schulkontext wird der Schulleitung eine wichtige Rolle zugeschrieben. Zu ihren Aufgaben zählt die generelle Entwicklung einer gemeinsamen Nutzung von digitalen Medien im Sinne eines schulischen Medienkonzeptes. Ebenso ist sie jene Stelle, die finanzielle und personale Ressourcen zur Implementierung von Innovationen zur Verfügung stellt (Gerick, Drossel, und Eickelmann 2014).

Lehrpersonen beeinflussen die Nutzung von hybriden Lehrmitteln wesentlich (Eickelmann und Lorenz 2014; Schulze-Vorberg u.a. 2018). So konnte Petko (2012) zeigen, dass ausreichend Kompetenz und eine positive Einstellung der Lehrpersonen Bedingungen für die Nutzung von hybriden Lehrmitteln im Unterricht sind.

Die genannten Aspekte lassen sich als schulische Medienkompetenz zusammenfassen, welche die Voraussetzungen für die Nutzung digitaler Medien generell und hybrider Lehrmittel im Speziellen schaffen. Gleichzeitig wird die Entwicklung digitaler bzw. hybrider Lehrmittel als ein Ansatz zur Förderung ebendieser schulischen Medienkompetenz vorgeschlagen (Eickelmann 2017).

Bisherige Studien betrachten in erster Linie die digitale Infrastruktur und den Einsatz von Internet- und Officeanwendungen im Unterricht (Petko 2012; Schulze-Vorberg u.a. 2018; Eickelmann 2014). Untersuchungen, die sich mit der Einführung und Nutzung von hybriden Lehrmitteln im Schulkontext auseinandersetzen, fehlen bislang noch. An dieser Stelle setzt der Beitrag an. 


\subsection{Zum hybriden Lehrmittel «dis donc!»}

Das Französischlehrmittel «dis donc!» ${ }^{1}$ richtet sich am neuen kompetenzorientierten Lehrplan 21 (Deutschschweizer Erziehungsdirektoren-Konferenz 2016) aus und unterstützt Französisch als zweite Fremdsprache ab der 5. Klasse (M. Keller u.a. 2012; Egli u.a. 2017). Es basiert unter anderem auf der sprachdidaktischen Weiterentwicklung ${ }^{2}$ der Aufgabenorientierung (Thonhauser 2016). Die Aufgaben sind mit authentischem Inputmaterial aus der Lebenswelt der Schülerinnen und Schüler verknüpft, das nicht oder nur geringfügig didaktisiert wurde.

Das Französischlehrmittel wurde als hybrides Lehrmittel konzipiert und besteht aus traditionellen gedruckten Lehrwerksteilen wie Arbeitsbuch und Nachschlagewerk für Schülerinnen und Schüler und einem Kommentar für Lehrpersonen. Zu jedem Kapitel (Unité) stehen Tests zur Verfügung. Integraler Teil des Französischlehrmittels ist eine digitale Lernplattform für Schülerinnen und Schüler und Lehrpersonen. Die dort zur Verfügung gestellten digitalen Elemente lassen sich in instruktional und konstruktiv orientierte Medien unterscheiden (Petko 2010; Totter und Wolfer 2016). Die instruktional orientierten digitalen Übungen (sog. Pour s'entraîner) dienen zum Durcharbeiten und Vertiefen der Inhalte. Es werden verschiedene Formate wie Multiple Choice, Drag \& Drop, Hotspot, Ordnen, Lückentext, Drop Down (Szerszeń 2014) eingesetzt. Audios in Form von Hörtexten, Liedern, Playbacks und damit verbundene Hörund Sprechübungen unterstützen die Lautschulung der Schülerinnen und Schüler. Zum Lernen von Vokabeln erfolgt eine Anbindung an Quizlet ${ }^{3}$. Mit dem Bilan électronique (formatives Assessment) können die Schülerinnen und Schüler am Ende jedes Kapitels das Erreichen der Lernziele mittels Selbstevaluation prüfen.

Das authentische Inputmaterial z.B. in Form von Videoclips steht den Schülerinnen und Schülern ebenfalls auf der digitalen Lernplattform zur Verfügung. Die digitalen Elemente sind mit Texten und Bildern im Arbeitsbuch verlinkt. Individuelle und kollektive kommunikative Aufgaben sind so gestaltet, dass zur Bearbeitung konstruktiv orientierte Medien wie offene

1 https://www.lmvz.ch/schule/dis-donc

2 Eine ausführliche Darstellung der Umsetzung der didaktischen Innovationen des Lehrmittels findet sich unter Totter u.a. (2019).

3 https://quizlet.com/de 
und multifunktionale Softwaretools (Weblogs, Wikis, Mindmaps etc.) oder allgemeine Office-Anwendungen verwendet werden können.

Durch das Erarbeiten und Üben der zur Verfügung gestellten Angebote und Aufgaben werden schrittweise Kompetenzen für die Bewältigung einer kommunikativen Schlussaufgabe aufgebaut. Diese Schlussaufgabe am Ende jedes Lernzyklus soll die Kompetenzentwicklungen sichtbar machen und spiegelt in vereinfachter Form den Input wider. So sollen die Schülerinnen und Schüler z.B. als Schlussaufgabe einen eigenen Weblog über ihre Freizeitbeschäftigungen erstellen.

$\mathrm{Zu}$ Beginn des Schuljahrs 2017/2018 wurde das neue hybride Französischlehrmittel «dis donc!» flächendeckend in der 5. Klasse obligatorisch an Primarschulen des Kantons Zürich eingeführt.

\section{Forschungsfragen}

Im Rahmen eines Forschungsprojektes des Zentrums für Schulentwicklung der Pädagogischen Hochschule Zürich wurde die Einführung des hybriden Lehrmittels im Kanton Zürich untersucht (Totter u.a. 2019). Ausgehend von den theoretischen Überlegungen werden in diesem Beitrag die Praktiken und Erfahrungen, die mit der Einführung und Nutzung eines hybriden Lehrmittels im Schulkontext einhergehen, untersucht. Ziel dabei ist es, Aufschluss über die Möglichkeiten und Herausforderungen des Einsatzes insbesondere der digitalen Elemente eines hybriden Lehrmittels zu erlangen. Im Zentrum stehen dabei folgende Forschungsfragen:

- Wie nutzen Schülerinnen und Schüler und Lehrpersonen die digitalen Elemente des hybriden Lehrmittels?

- Welche Möglichkeiten und Herausforderungen finden sich in Schulen zum Einsatz des hybriden Lehrmittels?

\section{Methodisches Vorgehen und Stichprobe}

Zur Beantwortung der Fragestellungen wurden in drei Primarschulen explorative Fallstudien durchgeführt. Das Vorgehen basiert auf einem Mixed-Methods-Ansatz, entsprechend dem konvergierenden Design nach Creswell und Plano Clark (2018). Die Datenerhebung erfolgte im Herbst 2017 und im Juni 2018. 
In der ersten Erhebungswelle wurden die drei Schulleitenden sowie fünf Französischlehrpersonen interviewt. In der zweiten Erhebungswelle wurden vier Gruppeninterviews mit jeweils acht bis zehn Schülerinnen und Schülern und vier Einzelinterviews mit den Französischlehrpersonen geführt. Zusätzlich nahmen alle Schülerinnen und Schüler der Klassen an einer schriftlichen Befragung teil.

In den zwölf leitfadengestützten Einzelinterviews mit Schulleitungen (zwei Frauen und ein Mann) und Lehrpersonen (drei Frauen und zwei Männer) wurde unter anderem gefragt, wie die Einführung des hybriden Lehrmittels in der Schule thematisiert wurde und welche Unterstützung die Lehrpersonen erhielten. Es wurde nach dem Einsatz der digitalen Lernplattform gefragt und den genutzten digitalen Elementen des hybriden Lehrmittels. Was wurde wo und wie gemacht und welche anderen digitalen Medien wurden allenfalls genutzt. Die digitale Infrastruktur der Schule wurde thematisiert; auch die Umsetzung sprachdidaktischer Weiterentwicklungen wurde angesprochen.

In den vier leitfadengestützten Gruppeninterviews mit Schülerinnen und Schülern wurde das Gefallen und die Nutzung der verschiedenen Lernangebote besprochen; insbesondere das Lernen am Computer. Stark angelehnt an das Gruppeninterview erfolgte eine schriftliche Befragung aller Schülerinnen und Schüler der 5. Klassen (N=78, 40 Mädchen, 35 Jungen, 3 keine Angaben, im Alter zwischen 11 und 12 Jahren) mittels Fragebogen. Auf einer vierteiligen Antwortskala konnten die Schülerinnen und Schüler angeben, wie sehr sie Aussagen zustimmen (Gefallen und Nutzung der Lernangebote, Verständnis des authentischen Inputmaterials, Einschätzung des Schwierigkeitsgrades der Aufgaben, Selbstwirksamkeit).

Die Interviewdaten wurden inhaltsanalytisch (Kuckartz 2014) ausgewertet. In einem ersten Schritt wurde das Interviewmaterial vollständig codiert und induktive Kategorien erstellt. In einem zweiten Schritt wurden diese Kategorien zu Hauptkategorien zusammengefasst: Dabei wurde unterschieden zwischen der Nutzung der digitalen Elemente durch die Schülerinnen und Schüler zu Hause und im Unterricht und der Nutzung der digitalen Elemente durch die Lehrpersonen zur Vorbereitung und im Unterricht. Die Möglichkeiten und Herausforderungen des Einsatzes der digitalen Elemente des hybriden Lehrmittels im Schulkontext wurde unter 
besonderer Berücksichtigung der schulischen Medienkompetenz im Sinne der digitalen Infrastruktur, der Rolle der Schulleitung und der Kompetenz und Einstellung der Lehrpersonen (vgl. Kapitel 1) zusammengefasst. Fragebogendaten der Schülerinnen und Schüler wurden mittels deskriptiver Statistik ausgewertet.

\section{Ergebnisse}

Im Folgenden werden die Ergebnisse präsentiert. Zunächst wird jede der drei Fallschulen kurz vorgestellt. Danach wird ausgehend von den Fragestellungen beschrieben,

1. wie sich die Nutzung der digitalen Elemente des Lehrmittels gestaltet;

2. welche Möglichkeiten und

3. welche Herausforderungen von der Schulleitung, den Lehrpersonen und Schülerinnen und Schülern wahrgenommen werden.

Abschliessend folgt eine Zusammenfassung der Ergebnisse über die Schulen hinweg.

\subsection{Fallschule 1}

Bei der untersuchten Schule handelte es sich um eine kleine Primarschule in ländlicher Umgebung im Kanton Zürich mit sieben Schulklassen (1. bis 6. Schulstufe) und zwei Kindergärten. 160 Schülerinnen und Schüler besuchten zum Zeitpunkt der Untersuchung die Schule und es gab eine 5 . Klasse, die von einer Klassenlehrperson in Französisch unterrichtet wurde. Die Lehrperson hatte fünf Jahre Unterrichtserfahrung.

Die Frage nach der Nutzung der digitalen Elemente des hybriden Französischlehrmittels durch die Schülerinnen und Schüler und der Lehrperson lässt sich wie folgt beantworten:

Sowohl die Schülerinnen und Schüler als auch die Lehrperson gaben an, dass die Schülerinnen und Schüler die instruktional orientierten digitalen Übungen (Pour s'entraîner) zur Prüfungsvorbereitung und zur Bearbeitung von Hausaufgaben zu Hause nutzten. Ebenfalls wurden die Audios (insbesondere die Sprech- und Hörübungen) und das formative 
Assessment (Bilan électronique) genutzt im Sinne der Übungs- und Kontrollfunktion. Besonders war, dass die Schülerinnen und Schüler neben dem hybriden Lehrmittel auch andere digitale Medien nutzten, z.B. eine Klassenplattform, ein Online-Wörterbuch und YouTube.

Die digitalen Elemente unterstützen die Steuerung im Unterricht. Die Lehrperson setzte Lieder zum Einstieg, Videoclips und Hörtexte zwischendurch bewusst zur Abwechslung mit anderen Unterrichtsformen ein. Schülerinnen und Schüler konnten gegen Ende der Stunde, wenn sie mit den Aufgaben im Buch fertig waren, die digitalen Elemente nutzen:

«Die konnten am Computer nochmals üben oder eine zusätzliche Aufgabe da noch lösen». (Zitat $L P 1, t 1,177)$.

Diese vielfältige Nutzung wurde in einer anderen Aussage nochmals von der Lehrperson bestätigt:

«Ja, die brauch ich, also ich selber brauch sie sicher jede Lektion eigentlich, um etwas abzuspielen oder zu zeigen» (Zitat $\mathrm{LP} 1, \mathrm{t} 1,127)$.

Auch wies die Lehrperson darauf hin, dass die Schülerinnen und Schüler das formative Assessment (Bilan électronique) immer in der letzten Stunde vor der Prüfung im Unterricht durcharbeiteten. Die Schülerinnen und Schüler nutzten diese Möglichkeit zur Standortbestimmung, also um zu erfahren, wie sie sich individuell auf die Prüfung vorbereiten sollten. Neben dem hybriden Lehrmittel hatte die Lehrperson eine Klassenplattform eingerichtet in Form einer Linksammlung, auf welche die Schülerinnen und Schüler ebenfalls zugreifen konnten.

Die Möglichkeiten, die sich beim Einsatz des hybriden Lehrmittels, insbesondere der Nutzung der digitalen Elemente ergeben, wurden wie folgt beschrieben: Die Lehrperson empfand den Einsatz des hybriden Lehrmittels als eine Bereicherung. Sie setzte die instruktional orientierten digitalen Übungen gezielt für Hausaufgaben ein. Schülerinnen und Schüler schätzten das hybride Lehrmittel sehr, auch weil es das erste Lehrmittel dieser Art ist, und die Angebote der digitalen Lernplattform mit dem Arbeitsbuch verlinkt sind. Die Lehrperson sah für die Schülerinnen und Schüler einen Mehrwert insbesondere dahingehend, dass die Schülerinnen und Schüler sich «etwas auch anhören, [...] es wird dann grad korrigiert und so» (Zitat LP1, t2, 209). 
Der Einsatz des hybriden Lehrmittels ging mit einigen Herausforderungen einher. Sowohl die Schulleitung als auch die Lehrperson gaben an, dass der Einsatz im Unterricht mit einem gewissen Aufwand verbunden war. So ging viel Zeit verloren, bis die digitale Infrastruktur (Beamer, Laptops, Tablets) im Klassenzimmer vorhanden war und alle Schülerinnen und Schüler eingeloggt waren. Mitunter überschätzte die Lehrperson die Anwendungskompetenz der Schülerinnen und Schüler. So konnten Schülerinnen und Schüler zwar Spiele spielen, aber Tastaturschreiben war für sie sehr schwierig.

Auch fehlte der Schule (noch) eine Person, die den pädagogischen ICTSupport übernimmt (PICTS). Die Schulleitung verwies auf die Schwierigkeit, Personen zu finden, die sich auf die Ausbildung dazu einlassen.

Als eine wesentliche Voraussetzung für den Einsatz hybrider Lehrmittel im Unterricht, die gleichzeitig eine grosse Herausforderung darstellte, wurde die Bereitstellung bzw. das Vorhandensein der digitalen Infrastruktur an der Schule genannt. Aus Sicht der Lehrperson war dabei zentral, genügend Geräte zu haben und dass die digitale Infrastruktur (Geräte und Verbindungen) funktioniert. Für die Schulleitung war es wichtig, dass die «Schule im richtigen Moment aufgesprungen ist» (Zitat SL1, 134) und vor zwei Jahren begonnen hatte, in jedem Klassenzimmer Beamer zu installieren und mindestens sechs Laptops pro Klasse zur Verfügung zu stellen.

Eine weitere wichtige Voraussetzung war das Vorhandensein von Unterstützung zur Nutzung des hybriden Lehrmittels. In dieser Schule wurde dazu ein breites Spektrum genannt. So erfolgte die Unterstützung auf Schulebene (als zentrale Aufgabe der Schulleitung) durch die Ermöglichung des Besuchs von Weiterbildungen, die Bildung einer Informatikkommission und das Vorhandensein einer Begleitperson und einer informatikverantwortlichen Person. Die Lehrperson verwies noch auf die Bedeutung der gegenseitigen Unterstützung im Kollegium, konkret auf die Möglichkeit sich mit dem Stellenpartner bzw. der Stellenpartnerin auszutauschen.

Für die Schulleitung war auch wichtig, dass die Lehrpersonen die Bereitschaft zeigten, Medienkompetenz zu entwickeln. Ihr war bewusst, dass dies Zeit braucht:

«Die elektronischen Dinge, [...] die muss man ja selber auch mal ausprobieren zuerst. Das braucht Zeit, oder. So da braucht's schon grossen Einsatz» (Zitat SL1, 84). 
Dieser grosse Einsatz zeigte sich bei der Lehrperson auch darin, dass sie die Eltern miteinbezog und den Einsatz des hybriden Lehrmittels am Elternabend thematisierte.

\subsection{Fallschule 2}

Die Schule bestand aus 15 Klassen der 4. bis 6. Schulstufe, die zum Zeitpunkt der Untersuchung von rund 350 Schülerinnen und Schüler besucht wurde. Die Schule wurde seit 2017 in Form einer Co-Schulleitung geführt. Die Schülerinnen und Schüler der beiden untersuchten Klassen wurden von zwei Fachlehrpersonen (LP2 und LP3) in Französisch unterrichtet, die beide seit ca. 15 Jahren als Lehrpersonen tätig waren.

Bezüglich der Nutzung der digitalen Elemente des hybriden Lehrmittels wurde ebenfalls die Übungs- und Kontrollfunktion betont. Die Schülerinnen und Schüler berichteten, dass sie verschiedene digitale Elemente zur Prüfungsvorbereitung zu Hause nutzten. Eine Lehrperson erwähnte, dass sie den Schülerinnen und Schülern gezielt digitale Elemente als Hausaufgaben gab. Von den Schülerinnen und Schülern wurden vor allem die Audios hervorgehoben. Positiv bewertet wurde dabei, dass sie hören konnten, wie man Wörter ausspricht und sie durch das hybride Lehrmittel eine erweiterte Repräsentationsfunktion zur Verfügung hatten. Das formative Assessment (Bilan électronique) wurde primär im Unterricht bearbeitet. Die Umsetzung der kommunikativen Schlussaufgabe mit Hilfe von konstruktiv orientierten digitalen Medien wurde von den Schülerinnen und Schülern ebenfalls positiv wahrgenommen.

«Ähm ich fand es einfach sehr cool, dass wir am Schluss noch einen eigenen Blog schreiben konnten. Über unsere Hobbies» (Zitat SuS, 100).

Eine Lehrperson merkte an, dass die Schülerinnen und Schüler auch andere digitale Medien nutzten wie YouTube oder das Smartphone, um Vokabeln zu lernen. Sie war allerdings überrascht über die Rückmeldung der Eltern, die berichteten, dass die digitalen Elemente zu Hause nicht oft zum Einsatz kämen oder nur dann, wenn die Eltern die Schülerinnen und Schüler explizit dazu anregten. 
Auch an dieser Schule nutzten die Lehrpersonen die digitalen Elemente im Sinne der Steuerungsfunktion, zur Vorbereitung, als Unterrichtseinstieg oder zwischendurch; insbesondere Lieder, Videoclips und Hörtexte. Die Schülerinnen und Schüler nahmen das Arbeiten mit den digitalen Elementen auch als eine Art Belohnung wahr. Waren sie mit Aufgaben im Arbeitsbuch fertig, durften sie die instruktional orientierten digitalen Übungen (Pour s'entraîner) bearbeiten, «sonst darf man nicht» (Zitat SuS, 255). Die Lehrpersonen verwendeten das hybride Lehrmittel mehr oder weniger täglich und schätzten folgendes daran:

«..., dass ich von überall zugreifen kann, dass ich es nicht mit nachhause schleppen muss, in jedem Klassenzimmer einfach habe» (Zitat LP2, t1, 207).

Bezüglich der Möglichkeiten des Einsatzes hybrider Lehrmittel nahmen die Lehrpersonen und die Schulleitung das individuelle, selbstbestimmte Arbeiten der Schülerinnen und Schüler wahr.

«Aber die Schüler schätzen das extrem, dass sie allein an der OnlinePlattform arbeiten können» (Zitat LP2, t1, 121).

Positive Äusserungen kamen auch von den Schülerinnen und Schülern selbst. Sie schätzten das individuelle Arbeiten, sowohl zu Hause als auch im Unterricht, aber auch die Vielfalt der digitalen Elemente, die angeboten wurden, wie Vokabellernen mit Quizlet, das Erstellen eines Weblogs und die Videoclips.

Die Lehrpersonen sowie die Schulleitung dieser Schule sahen die Nutzung des hybriden Lehrmittels mit folgenden Herausforderungen verbunden. Es war ein gewisser Aufwand nötig, bis die Schülerinnen und Schüler wirklich arbeiten konnten. Bezüglich des Aufwands nahm eine Lehrperson einen Lerneffekt wahr. Am Ende des Schuljahrs loggten sich die Schülerinnen und Schüler im Vergleich zum Beginn schon viel schneller ein. Dennoch hing dies auch von den Lernenden ab.

«Je nach Konzentration der Schüler, oder was es für TYpen sind, dann brauchen sie mal länger und dann googlen sie doch noch $n$ bisschen rum» (Zitat LP2, t2, 49). 
Als kritisch erachteten die Lehrpersonen, dass sie nicht genau überblickten, woran die Schülerinnen und Schüler arbeiteten, vor allem bei den Hausaufgaben. Sie würden sich wünschen, «dass man als Lehrperson nachschauen kann, was haben die Kinder gearbeitet» (Zitat LP3, t1, 135).

Das Funktionieren der digitalen Infrastruktur war ebenfalls von zentraler Bedeutung. Vor allem die Schülerinnen und Schüler schilderten einige Situationen, in denen der Login nicht funktionierte, was vor allem zu Hause zu Problemen führte. In manchen Situationen lösten die Schülerinnen und Schüler das Problem, indem Login-Daten einer anderen Person verwendet wurden.

Eine ausreichende digitale Infrastruktur war laut den Lehrpersonen eine zentrale Voraussetzung für den Einsatz des hybriden Lehrmittels und gleichzeitig eine grosse Herausforderung. So fehle es an einem vollständigen Klassensatz an Tablets, um flexibel arbeiten zu können. Da diese zunehmend auch in anderen Fächern verwendet wurde, war die Verfügbarkeit nicht immer gegeben. Dies erschwerte den Lehrpersonen die Planung. Weitere Herausforderung waren:

«..., [dass] die Kopfhörer gehen, dass die alle ihren Login haben, [...] im Schulalltag doch noch , n grösseres Hindernis als man meinen sollte» (Zitat $L P 2, \mathrm{t} 1,121)$.

Aus Sicht der Schulleitung und einer Lehrperson war zudem das Vorhandensein von Unterstützung von grosser Bedeutung. Eine wichtige Rolle spielte dabei die informatikverantwortliche Person, die von den Lehrpersonen bei Problemen immer kontaktiert werden konnte. Die Schulleitung sah es als ihre Aufgabe, «Dinge zur Verfügung zu stellen» (Zitat SL2, 60). Für die Schulleitung war ausserdem relevant, dass die Schülerinnen und Schüler (noch) Medienkompetenz entwickeln (müssen). Auch an dieser Schule bezog eine Lehrperson die Eltern mit ein und besprach den Einsatz des hybriden Lehrmittels an einem Elternabend. 


\subsection{Fallschule 3}

Die Schule einer ländlichen Gemeinde wurde von rund 300 Schülerinnen und Schülern besucht, die sich auf zwölf Primarschulklassen (1. bis 6 . Schulstufe) und vier Kindergärten verteilten. Es gab zwei 5. Klassen, die von den Lehrpersonen (LP4 und LP5) als Klassenlehrpersonen in Französisch unterrichtet wurden. Eine Lehrperson unterrichtete seit mehr als 10 Jahren, die andere seit mehr als 25 Jahren.

Die digitalen Elemente wurden wie folgt genutzt: Von den Lehrpersonen wurden instruktional orientierte digitale Übungen (Pour s'entraîner) als Hausaufgaben gegeben. Die Schülerinnen und Schüler verwendeten die digitalen Elemente, um sich gezielt auf Prüfungen vorzubereiten oder Inhalte nachzubearbeiten, die sie noch nicht gut verstanden hatten. Das Nachsprechen, das Hören von Texten und Liedern und das Vokabellernen mit Quizlet wurden von den Schülerinnen und Schülern bewusst zum Üben und Kontrollieren verwendet und geschätzt. Die Nutzung der digitalen Elemente erfolgte gekoppelt mit dem Arbeitsbuch, wie eine Schülerin darlegte:

«Wir machen eigentlich meistens die Aufgaben, die wir im Buch auch gemacht haben» (Zitat SuS, 426).

Eine Lehrperson führte die Schülerinnen und Schüler im Unterricht ein, «wie sie zu Hause üben können am Computer» (Zitat LP5, t1, 103).

Im Unterricht wurde das hybride Lehrmittel in den zwei Klassen unterschiedlich verwendet. So wurden in einer Klasse zur Strukturierung des Unterrichts Videoclips und Hörtexte eingesetzt. In der anderen Klasse sah die Lehrperson die Nutzung der digitalen Elemente weniger innerhalb des Unterrichts und begründete dies damit, dass das Sprechen sonst zu kurz käme.

«Eben wie gesagt in der Schule während des Unterrichts arbeite ich sozusagen nicht am Computer mit ihnen, weil ich, ich möchte gerne, dass sie mit mir zusammen sprechen, dass ich vorspreche. Bei einem Computer ist sehr viel einfach nur (still) Arbeit und da fehlt einfach das das Sprechen» (Zitat LP5, t1, 159). 
Allerdings setzte die Lehrperson digitale Elemente für die synchrone Kommunikation ein. Sie liess die Schülerinnen und Schüler des Öfteren zu zweit arbeiten, was laut den Schülerinnen und Schülern Vorteile hatte.

«Wenn der eine nicht ganz draus kommt, kann der andere zum Beispiel, wenn er's wirklich weiss einmal ein bisschen einen Tipp geben» (Zitat SuS, 199).

Von Seiten der Schulleitung wurde betont, verschiedene Unterrichtsformen bewusst einzusetzen.

«... einfach wichtig, dass nicht ausschliesslich eine Art gemacht wird, aber die Gefahr ist ja klein, oder. Also wenn man dann alles nur noch digital macht, ist das so schlecht wie wenn man alles mit Werkstattunterricht oder alles mit Frontalunterricht, oder alles, ich denke der Mix, der macht das aus» (Zitat SL3, 118).

In dieser Schule nutzten die Lehrpersonen keine weiteren digitalen Medien.

Die Möglichkeiten der digitalen Elemente des hybriden Lehrmittels wurden von den Lehrpersonen vor allem im Hausaufgaben geben gesehen. Somit konnten sie auch das Problem einer unzureichenden digitalen Infrastruktur umgehen, wenn z.B. nicht ausreichend Computer in der Schule vorhanden waren. Laut den Lehrpersonen schätzten die Schülerinnen und Schüler die digitale Lernplattform sehr. Eine Lehrperson begründet dies wie folgt:

«Das geht ja so ein bisschen ins Individuelle, dass die Schüler selber dort Übungen machen können, genau, und alles eigentlich auch nochmal hören» (Zitat LP4, t2, 20).

Von den Schülerinnen und Schülern selbst kamen zahlreiche positive Kommentare zum hybriden Lehrmittel, wobei sie den Unterhaltungsfaktor hervorhoben, den das Arbeiten mit der digitalen Lernplattform mit sich brachte.

«Ja und bei dem, beim Computer ist auch lustig irgendwie die Tests, also die Tests zu machen und anderen Aufgaben» (Zitat SuS, 80). 
Die Schülerinnen und Schüler wiesen auch darauf hin, dass es für manche eine geeignetere Lernmöglichkeiten sei als für andere:

«Ich finde es einfach für die, die es wirklich so besser lernen können, ist es wirklich toll, wenn es das gibt» (Zitat SuS, 251).

Als Herausforderung beschrieb eine Lehrperson, dass sich der Einsatz des hybriden Lehrmittels lohnen müsse, was zum Beispiel nicht der Fall war, wenn nur eine digitale Aufgabe bearbeitet werden sollte und dafür allen Schülerinnen und Schüler Laptops bereitgestellt werden mussten. Von den Lehrpersonen wurde auch die fehlende Kontrolle darüber angesprochen, was die Schülerinnen und Schüler digital bearbeiteten, wobei ein pragmatischer Umgang gefunden wurde:

«Man kann's nicht kontrollieren, vielleicht ändert sich das mal. Man kann aber auch sagen, man muss nicht immer alles kontrollieren》 (Zitat LP4, t2. 58).

Die Lehrpersonen verwiesen auch kritisch darauf, dass die Schülerinnen und Schüler teilweise Zugriffe auf Programme wie z.B. bestimmte Internetbrowser benötigten, die sie zu Hause nicht unbedingt hatten, um die digitalen Elemente nutzen zu können. Bevor für Hausaufgaben die digitale Lernplattform genutzt werden konnte, musste zuerst geklärt werden, ob jede Schülerin bzw. jeder Schüler einen digitalen Zugang von zu Hause aus besitzt.

Von den Schülerinnen und Schülern wurde kritisch angemerkt, dass das hybride Lehrmittel nicht immer freundlich war und manche Funktionen nicht einwandfrei funktionierten.

«Nicht alle Seiten kann man draufdrücken [...]. Ja manche sind einfach leer und man kanns nicht mal anschauen» (Zitat SuS, 429).

Die digitale Infrastruktur an der Schule wurde als bereits recht gut beschrieben. Es gab Laptops und teilweise Klassensätze an IPads. Als explizites Ziel, welches spätestens in zwei Jahren erreicht werden sollte, nannte die Schulleitung die individuelle Ausstattung für jede Schülerin und jeden Schüler. Von der Schulleitung wurde die Medienkompetenz der Französischlehrpersonen bzw. des gesamten Teams so beschrieben, dass sie an 
das Arbeiten «mit digitalen Sachen» (Zitat SL3, 126) gewöhnt seien und auch Weiterbildungen zum Thema absolvierten. Auch in dieser Schule wurden die Eltern zum Umgang mit dem hybriden Lehrmittel von den Lehrpersonen am Elternabend informiert.

Abschliessend erfolgt eine Zusammenfassung der Ergebnisse über die drei Fallschulen hinweg. Bezüglich Nutzung zeigte sich, dass die Schülerinnen und Schüler aller Schulen und Klassen die digitale Lernplattform des hybriden Lehrmittels kontinuierlich zur Prüfungsvorbereitung und zur Bearbeitung von Hausaufgaben (zu Hause) verwendeten. Dabei wurden insbesondere die instruktional orientierten digitalen Übungen (sog. Pour s'entraîner) und die mit den Audios verbundenen Hör- und Sprechübungen bearbeitet.

Das formative Assessment (Bilan électronique) wurde ebenfalls regelmässig genutzt; angeleitet im Unterricht, als Hausaufgabe oder selbstbestimmt durch die Schülerinnen und Schüler im Rahmen der Prüfungsvorbereitung.

Übereinstimmung fand sich auch bezüglich der Verwendung von Audios (Lieder und Hörtexte) und Videoclips im Unterricht als Einstieg und zwischendurch.

In einigen Klassen durften die Schülerinnen und Schüler die digitale Lernplattform am Ende der Stunde nutzen, nachdem sie mit ihren Aufgaben aus dem Arbeitsbuch fertig waren.

Aus der schriftlichen Befragung aller Schülerinnen und Schüler ging hervor, dass das Arbeitsbuch (Aufgaben lösen und Nachlesen) des hybriden Lehrmittels die erste Wahl war, um Französisch zu lernen (siehe Abbildung 1). Knapp 20\% der Schülerinnen und Schüler gaben an, dafür auch die verschiedenen Elemente der digitalen Lernplattform sehr oft zu nutzen. 


\section{Was nutzt du zum Französischlernen?}

Ich löse Aufgaben im Arbeitsbuch.

Ich lese im Arbeitsbuch nach.

Ich löse «Pour s'entraîner» auf der digitalen Lernplattform.

Ich schau mir «Bilan électronique» auf der digitalen Lernplattform an.

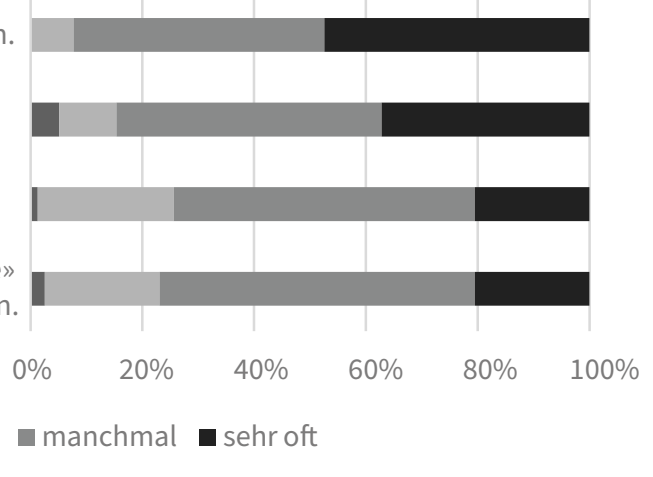

Abb. 1.: Nutzung der Lernangebote zum Französischlernen durch Schülerinnen und Schüler ( $N=78$, 4-teilige Antwortskala).

Hinsichtlich der Möglichkeiten standen alle Befragte dem hybriden Lehrmittel sehr positiv gegenüber. Die Schülerinnen und Schüler und die Lehrpersonen gaben in den Interviews an, die digitalen Elemente des hybriden Lehrmittels sehr zu schätzen. Dies wurde in der schriftlichen Befragung von den Schülerinnen und Schüler bestätigt. Über alle Klassen hinweg gaben 73.1\% der befragten Schülerinnen und Schülern $(\mathrm{N}=78)$ an, dass ihnen das Lernen mit der digitalen Lernplattform sehr gut gefiel.

Konkret nannten die Schülerinnen und Schüler das individuelle Arbeiten und die Vielfalt des digitalen Angebots als besonders positive Aspekte. Instruktional orientierte digitale Übungen (Pour s'entraîner) als Hausaufgabe geben zu können, wurde von einem Teil der Lehrpersonen als gute Möglichkeit gesehen, das Üben zu unterstützen.

Im Zusammenhang mit der sprachdidaktischen Aufgabenorientierung wurde in allen Gruppeninterviews von den Schülerinnen und Schülern die Umsetzung von kommunikativen Schlussaufgaben mithilfe von konstruktiv orientierten digitalen Medien positiv erwähnt, insbesondere das Erstellen eines Weblogs.

Alle Schulleitungen und Lehrpersonen nannten die Bereitstellung und das Funktionieren der digitalen Infrastruktur in den Schulen als die zentrale Herausforderung. Obwohl eine gewisse digitale Infrastruktur vorhanden war, betonten die Schulleitungen, diese in den nächsten Jahren noch 
weiter auszubauen zu wollen. Ein weiterer Aspekt, der in allen Einzelinterviews thematisiert wurde, war der mit der Nutzung im Unterricht verbundene Aufwand. Zum einen musste ein Teil der digitalen Infrastruktur vorab reserviert und aus anderen Räumen der Schule ins Klassenzimmer geholt werden. Zum anderen brauchten die Schülerinnen und Schüler viel Zeit, um sich auf der digitalen Lernplattform einzuloggen, was sich negativ auf die verbleibende Unterrichtszeit zur inhaltlichen Bearbeitung auswirkte.

Die Unterstützung der Lehrpersonen durch die Schulleitung ging kaum über die Bereitstellung von personellen und finanziellen Ressourcen hinaus. Eine wichtige Funktion übernahm die informatikverantwortliche Person als Anlaufstelle bei Problemen. Schulleitende zweier Schulen verlangten von ihren Lehrpersonen eine grundsätzliche Bereitschaft zur Entwicklung einer Medienkompetenz. Die Lehrpersonen selbst erwähnten diese nicht. Für die Öffnung der Nutzung hybrider Lehrmittel in den ausserschulischen Lernbereich (zu Hause) wurden in allen Schulen die Eltern miteinbezogen.

\section{Diskussion und Ausblick}

Aus den Ergebnissen dieser Untersuchung wurde deutlich, dass ein hybrides Lehrmittel im ersten Jahr der Einführung von allen Beteiligten durchwegs positiv wahrgenommen wurde. Im Folgenden wird diskutiert, wie sich durch eine solche Innovation die Nutzung und Funktion eines Lehrmittels verändert. In den Fallschulen wurden die auf der digitalen Lernplattform zur Verfügung gestellten Audios (Lieder, Hörtexte) und Videoclips von den Lehrpersonen zur Strukturierung des Unterrichts genutzt. Durch die multimedial und interaktiv aufbereiteten Informationen und Inhalte konnte die Repräsentationsfunktion des Lehrmittels erweitert werden (Rodríguez Regueira und Rodríguez Rodríguez 2015; Döbeli Honegger, Hielscher, und Hartmann 2018). Schülerinnen und Schüler nutzten die in den Audios zur Verfügung gestellten Hör- und Sprechübungen auch zu Hause für die Lautschulung. Sprachdidaktische Innovationen wie z.B. die Aufgabenorientierung erfuhren neue Möglichkeiten. Durch authentisches und aktuelles Inputmaterial konnte an die Lebenswelt der Schülerinnen und Schüler 
angeknüpft werden (Thonhauser 2016). Kommunikative Schlussaufgaben, wie das Erstellen eines Weblogs konnten gemeinsam und mittels digitaler Produkte gestaltet werden.

Die Differenzierungsfunktion wurde ebenfalls erweitert. Auf der digitalen Lernplattform standen vielfältige und umfangreiche Lernangebote zur Verfügung. Schülerinnen und Schüler hatten die Möglichkeit, Aufgaben und Übungen entsprechend ihrer individuellen Möglichkeiten bzw. Bedürfnisse auszuwählen (Schaumburg 2015). Sie nutzten diese Funktion bewusst, primär im ausserschulischen Lernbereich (zu Hause) (SchulzeVorberg u.a. 2018) zur Prüfungsvorbereitung und um Inhalte nachzubearbeiten.

Die Übungs- und Kontrollfunktion wurde durch verschiedene digitale Elemente in Richtung individueller Lernunterstützung erweitert. Die digitalen formativen Assessments (Bilan électronique) wurden von den Schülerinnen und Schülern selbstreguliert bearbeitet und gaben unmittelbare Rückmeldung über Lernfortschritte (Döbeli Honegger, Hielscher, und Hartmann 2018). Ebenso wurden Vokabel mit Quizlet gelernt bzw. kontolliert und die Hör- und Sprechübungen genutzt.

Der bewusste Einsatz der digitalen Elemente von den Lehrpersonen als Belohnung für die Schülerinnen und Schüler ist im Zusammenhang mit der geforderten Motivierungsfunktion von Lehrmitteln kritisch zu hinterfragen. Dass die Schülerinnen und Schüler Spass haben und dass ihnen das Lernen mit der digitalen Lernplattform sehr gut gefällt, bestätigte grundsätzlich das Motivierungspotential hybrider Lehrmittel (Schaumburg 2015).

Bezüglich des Potentials zur impliziten Schulung der Anwendungskompetenz durch die Nutzung von Office-Anwendungen ergaben sich Hinweise darauf, dass Lehrpersonen mitunter von zu hohen Erwartungen an die Schülerinnen und Schüler als «digital natives» ausgehen (Petko, Honegger, und Prasse 2018; Schaumburg 2015). Insbesondere die Kompetenz des Tastaturschreibens im Fremdsprachenunterricht überforderte die Schülerinnen und Schüler und müsste in der 5 . Klasse gefördert werden. Hinweise zur Schulung der Medienkompetenz (Petko, Honegger, und Prasse 2018), die über die Anwendung bzw. Mediennutzung hinaus gingen in Richtung Medienkritik, Medienkunde oder Mediengestaltung fanden sich in den Fallstudien keine. 
Bezüglich Herausforderungen bei der Einführung und Nutzung eines hybriden Lehrmittels, zeigte sich folgendes Bild. In allen Schulen wurden darauf hingewiesen, dass die aktuelle digitale Infrastruktur der gewünschten und zum Teil notwendigen Ausstattung hinterherhinkt. Inwiefern sich in den letzten Jahren eine Verbesserung der Ausstattung ergab bzw. sich in nächster Zeit aufgrund des Bildungsratsbeschlusse «ICT an Zürcher Volksschulen 2022» (Bildungsdirektion Kanton Zürich 2016) ergeben wird, ist zu prüfen. Der schulische Einsatz digitaler Medien sollte durch die Einführung hybrider Lehrmittel in nächster Zeit an den OECD-Durchschnitt angeglichen werden.

Schulleitungen fühlten sich primär verantwortlich für die Bereitstellung der digitalen Infrastruktur und finanzieller und personaler Ressourcen. Die Entwicklung eines gemeinsamen schulischen Medienkonzeptes, der aktive Austausch über die mit dem Einsatz des hybriden Lehrmittels einhergehenden Neuerungen oder die Teilnahme der Schulleitung an einem damit verbundenen Lernprozess im Sinne einer «Gatekeeper» Funktion (Fullan 2007; Gerick, Drossel, und Eickelmann 2014) für schulische Innovationen wurde von keiner Person thematisiert.

Die Ergebnisse weisen darauf hin, dass sich durch hybride Lehrmittel und dem damit verbundenen Einsatz von digitalen Medien die Funktionen von Lehrmitteln verändern. Damit sich das Potenzial von hybriden Lehrmitteln entfalten kann, braucht es allerdings das Bewusstsein bei Schulleitungen und Lehrpersonen die Einführung eines solchen Lehrmittels als Zusammenspiel von Schul- und Unterrichtsentwicklung zu gestalten (Totter u.a. 2019). Die Einführung von hybriden Lehrmitteln kann durchaus als Anlass genommen werden, das schulische Medienkonzept bewusst zu hinterfragen und die Entwicklung einer umfassenden Medienkompetenz der Lehrpersonen und Schülerinnen und Schüler als überfachliche Kompetenz vieler Fächern zu gestalten.

\section{Literatur}

Astleitner, Hermann. 2012. "Schulbuch und neue Medien im Unterricht: Theorie und empirische Forschung zur Hybridisierung und Komplementarität». In Schulbücher im Fokus. Nutzungen, Wirkungen und Evaluation, herausgegeben von Jörg Doll, Frank Keno, Detlef Fickermann, und Knut Schwippert, 101-11. Münster: Waxmann. 
Bildungsdirektion Kanton Zürich. 2016. «ICT an Zürcher Volksschulen 2022, BBR Nr. 24/2016». Zürich: Bildungsrat.

Creswell, John W., und Vicki L. Plano Clark. 2018. Designing and conducting mixed methods research. Third Edition. Los Angeles: SAGE.

Deutschschweizer Erziehungsdirektoren-Konferenz. 2016. Lehrplan 21. Gesamtausgabe. Luzern: Deutschschweizer Erziehungsdirektoren-Konferenz (DEDK). www.lehrplan.ch.

Döbeli Honegger, Beat, Michael Hielscher, und Werner Hartmann. 2018. «Lehrmittel in einer digitalen Welt. Expertenbericht im Auftrag der Interkantonalen Lehrmittelzentrale (ilz)». Interkantonale Lehrmittelzentrale (ilz).

Egli, Isabelle, Eliane Hueber, Marlies Keller-Lee, Christine Rast, Catherine Sachser, und Barbara Wolfer. 2017. dis donc! 5. Herausgegeben von Lehrmittelverlag Zürich, Lehrmittelverlag St. Gallen, und Interkantonale Lehrmittelzentrale. Zürich, St Gallen: Lehrmittelverlag.

Eickelmann, Birgit, Hrsg. 2014. Grundschule in der digitalen Gesellschaft: Befunde aus den Schulleistungsstudien IGLU und TIMSS 2011. Münster: Waxmann.

Eickelmann, Birgit. 2017. «Schulische Medienkompetenzförderung». In Medienkompetenz. Herausforderung für Politik, politische Bildung und Medienbildung, herausgegeben von Harald Gapski, Monika Oberle, und Walter Staufer, 14654. Bonn: Bundeszentrale für politische Bildung.

Eickelmann, Birgit, und Ramona Lorenz. 2014. «Wie schätzen Grundschullehrerinnen und -lehrer den Stellenwert digitaler Medien ein?» In Grundschule in der digitalen Gesellschaft: Befunde aus den Schulleistungsstudien IGLU und TIMSS 2011, herausgegeben von Birgit Eickelmann, 49-57. Münster: Waxmann.

Fuchs, Eckhardt, Inga Niehaus, und Almut Stoletzki. 2014. Das Schulbuch in der Forschung. Analysen und Empfehlungen für die Bildungspraxis. Göttingen: V\&R unipress $\mathrm{GmbH}$.

Fullan, Michael. 2007. New Meaning of Educational Change. 4. Aufl. New York \& London: Teachers College Press.

Gerick, Julia, Kerstin Drossel, und Birgit Eickelmann. 2014. «Zur Rolle der Schulleitung bei der Integration digitaler Medien in Grundschulen». In Grundschule in der digitalen Gesellschaft: Befunde aus den Schulleistungsstudien IGLU und TIMSS 2011, herausgegeben von Birgit Eickelmann, 35-47. Münster: Waxmann.

Gerick, Julia, Mario Vennemann, Ramona Lorenz, und Birgit Eickelmann. 2014. «Schulische Ausstattung mit digitalen Medien in der Grundschule». In Grundschule in der digitalen Gesellschaft: Befunde aus den Schulleistungsstudien IGLU und TIMSS 2011, herausgegeben von Birgit Eickelmann, 19-34. Münster: Waxmann.

Hacker, Hartmut, Hrsg. 1980. Das Schulbuch: Funktion u. Verwendung im Unterricht. Studientexte zur Grundschuldidaktik. Bad Heilbrunn/Obb: Klinkhardt.

Heitzmann, Anni, und Alois Niggli. 2010. «Lehrmittel - ihre Bedeutung für Bildungsprozesse und die Lehrerbildung». Beiträge zur Lehrerbildung 28 (1): 6-19. 
Hiller, Andreas. 2012. Das Schulbuch zwischen Internet und Bildungspolitik: Konsequenzen für das Schulbuch als Leitmedium und die Rolle des Staates in der Schulbildung. Marburg: Tectum Wissenschaftsverlag.

Keller, Marlies, Barbara Wolfer, Peter Klee, Michael Eisner, und Brigitta Gubler. 2012. «Konzept für die Entwicklung eines neuen interkantonalen Französischlehrmittels für die 5.-9. Klasse. Fassung vom 28. August 2012». Zürich.

Konsortium PISA.ch. 2019. PISA 2018: Schülerinnen und Schüler der Schweiz im internationalen Vergleich. Bern und Genf: SBFI/EDK und Konsortium PISA.ch. https:/pisa.educa.ch/sites/default/files/uploads/2019/12/pisa2018_de.pdf.

Kuckartz, Udo. 2014. Qualitative Inhaltsanalyse. Methoden, Praxis, Computerunterstützung. 2. Aufl. Weinheim: Beltz Juventa.

Macgilchrist, Felicitas. 2017. «Digitale Schulbücher. Chancen und Herausforderungen für den politischen Fachunterricht.»In Medienkompetenz. Herausforderung für Politik, politische Bildung und Medienbildung, herausgegeben von Harald Gapski, Monika Oberle, und Walter Staufer, 226-34. Schriftenreihe / Bundeszentrale für Politische Bildung. 101ll. Bonn: Bundeszentrale für politische Bildung.

Mader, Sabine, und Alois Bachinger. 2017. «Klassisches Schulbuch meets MeBook». In Schule neu denken und medial gestalten, herausgegeben von Nina Grünberger, Klaus Himpsl-Gutermann, Petra Szucsich, Gerhard Brandhofer, Edmund Huditz, und Michael Steiner, 459-74. Glückstadt: vwh-Verlag.

Matthes, Eva, Sylvia Schütze, und Werner Wiater, Hrsg. 2013. Digitale Bildungsmedien im Unterricht. Klinkhardt Forschung. Bad Heilbrunn: Klinkhardt.

Petko, Dominik. 2010. «Neue Medien - Neue Lehrmittel? Potenziale und Herausforderungen bei der Entwicklung digitaler Lehr- und Lernmedien». Beiträge zur Lehrerinnen- und Lehrerbildung 28 (1): 42-52.

Petko, Dominik. 2012. "Hemmende und förderliche Faktoren des Einsatzes digitaler Medien im Unterricht: Empirische Befunde und forschungsmethodische Probleme». In Jahrbuch Medienpädagogik 9, herausgegeben von Renate SchulzZander, Birgit Eickelmann, Heinz Moser, Horst Niesyto, und Petra Grell, 2950. Wiesbaden: VS Verlag für Sozialwissenschaften.

Petko, Dominik, Beat Döbeli Honegger, und Doreen Prasse. 2018. «Digitale Transformation in Bildung und Schule: Facetten, Entwicklungslinien und Herausforderungen für die Lehrerinnen- und Lehrerbildung». Beiträge zur Lehrerinnen- und Lehrerbildung 36 (2): 157-75.

Rodríguez Regueira, Nerea, und Jesús Rodríguez Rodríguez. 2015. «The digital textbook. A look at the current state of the art». In Digital Textbooks. What's new? herausgegeben von Jesús Rodríguez Rodríguez, Eric Bruillard, und Mike Horsley, 2015. Aufl. Santiago de Compostela: Santiago de Compostela: IARTEM/Servizo de Publicacións USC. 
Sankofi, Martin. 2017. "E-Books in Action - Entwicklungen rund um das digitale Schulbuch». In Schule neu denken und medial gestalten, herausgegeben von Nina Grünberger, Klaus Himpsl-Gutermann, Petra Szucsich, Gerhard Brandhofer, Edmund Huditz, und Michael Steiner, 459-74. Didaktik. 18. Glückstadt: Werner Hülsbusch.

Schaumburg, Heike. 2015. «Chancen und Risiken digitaler Medien in der Schule». Bertelsmann Stiftung.

Schmidt, Romina. 2019. Zur Bedeutung didaktischer Artefakte im Rechtschreibunterricht: Eine qualitative Studie zum Gebrauch von Lehr-Lern-Materialien durch Lehrpersonen. Wiesbaden: Springer Fachmdien.

Schulze-Vorberg, Lukas, S. Franziska C. Wenzel, Claudia Bremer, und Holger Horz. 2018. «Die Öffnung von (Lern-)Räumen in Schule und Unterricht durch den Einsatz digitaler Medien. Der Einfluss von Computereinstellung, -ängstlichkeit und Lehrhaltung auf die digitale Mediennutzung von Lehrkräften». In Jahrbuch Medienpädagogik 14, herausgegeben von Manuela Pietraß, Johannes Fromme, Petra Grell, und Theo Hug, 215-36. Wiesbaden: Springer Fachmedien.

Szerszeń, Pawel. 2014. «Aktuelle Tendenzen im computerunterstützten (Fach-) Fremdsprachenunterricht». Zeitschrift für Interkulturellen Fremdsprachenunterricht 19 (1).

Thonhauser, Ingo. 2016. «Was macht gute Aufgaben für den Fremdsprachenunterricht aus? Charakteristik guter Aufgaben und Einsichten aus der Unterrichtsbeobachtung». In Aufgaben als Schlüssel zur Kompetenz: didaktische Herausforderungen, wissenschaftliche Zugänge und empirische Befunde, herausgegeben von Stefan Keller, Christian Reintjes, und Viktor Abt, 179-96. Münster: Waxmann.

Totter, Alexandra, Daniela Müller-Kuhn, Enikö Zala-Mezö, und Simona Marti. 2019. «Schulbuch und Innovation? Die Einführung eines neuen Lehrmittels als (kein) Anlass zum Innovationstransfer». DDS - Die Deutsche Schule 111 (3): 294-309.

Totter, Alexandra, und Barbara Wolfer. 2016. «Formative Evaluation von digitalen Lehr- und Lernmedien am Beispiel des neuen Französischlehrmittels dis donc!» Medienimpulse 54 (3): 1-20. https://medienimpulse.at/article/view/ mig67. 